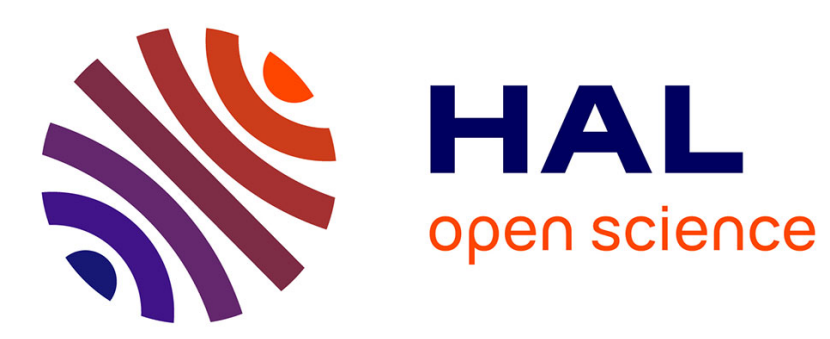

\title{
Convex Hierarchical Analysis for the Performances of Uncertain Large-Scale Systems
}

\author{
M Dinh, Anton Korniienko, Gérard Scorletti
}

\section{To cite this version:}

M Dinh, Anton Korniienko, Gérard Scorletti. Convex Hierarchical Analysis for the Performances of Uncertain Large-Scale Systems. 53rd IEEE CDC, Dec 2014, Los Angeles, CA, United States. 10.1109/CDC.2014.7040325 . hal-01089428v2

\section{HAL Id: hal-01089428 \\ https://hal.science/hal-01089428v2}

Submitted on 29 Jul 2015

HAL is a multi-disciplinary open access archive for the deposit and dissemination of scientific research documents, whether they are published or not. The documents may come from teaching and research institutions in France or abroad, or from public or private research centers.
L'archive ouverte pluridisciplinaire HAL, est destinée au dépôt et à la diffusion de documents scientifiques de niveau recherche, publiés ou non, émanant des établissements d'enseignement et de recherche français ou étrangers, des laboratoires publics ou privés. 


\title{
Convex Hierarchical Analysis for the Performances of Uncertain Large-Scale Systems
}

\author{
M. Dinh, A. Korniienko and G. Scorletti
}

\begin{abstract}
The performance analysis of uncertain large-scale systems is under consideration. It is performed via a hierarchical modeling and analysis of the systems thanks to the recursive application of a propagation of dissipativity properties result. In contrast to an one-step approach such as (upper bound) $\mu$-analysis where computation time can be prohibitive for large-scale systems, the proposed method allows to set the trade-off between conservatism and computation time. The approach is used on a PLL network example and illustrates the new trade-off achieved.
\end{abstract}

\section{INTRODUCTION}

In the 80's-90's, $\mu$-analysis [7], [22] was developed to investigate the $\left(H_{\infty}\right)$ performance of Linear Time Invariant systems in the case of structured uncertainties. This approach is based on the computation of the structured singular value $\mu$ of frequency dependent complex matrices, which was proved to be NP-hard by [3]. Fortunately, lower bound and upper bounds on $\mu$ can be efficiently computed, the computation of the $\mu$ upper bound [8] allowing to guarantee a certain level of performance, with some conservatism. A major interest of the use of $\mu$ upper bound is to obtain a satisfying trade-off between the complexity of the computation (i.e. the efficiency) and the conservatism of the obtained result.

Nevertheless, even if the computation is efficient, the computation time can be important in the case of largescale uncertain systems. The purpose of the paper is to propose a robust analysis method with a trade-off between efficiency and conservatism adapted to large-scale systems. Our motivating application is the robustness analysis of Phase-Locked-Loop networks [11], [12], a challenging problem in Microelectronics.

In the case of large-scale systems with uncertainties defined by cones, [16] proposed to perform a hierarchical analysis using a propagation of conic uncertainty result. Unfortunately, its direct application to a (large-scale) system with structured uncertainties could lead to an overly conservative result. In this paper, we propose a new hierarchical analysis method which is adapted to the case of uncertain systems with structured uncertainties.

As in [16], a hierarchical system is modeled as a tree. Basically, the leaves of the tree are sub-systems that are interconnected with an interconnection matrix; the overall being a branch. In fact, this branch is itself viewed as a leave that is connected to another interconnection matrix, the overall being a new branch, and so on. Assuming

M. Dinh is with Unit Mathématique, Informatique \& Génomique Institut National de la Recherche Agronomique 78352 Jouy en Josas Cedex, Franc marc.dinhejouy.inra.fr

A. Korniienko and G. Scorletti are with Laboratoire Ampère Dpt. EEA of the Ecole Centrale de Lyon, Université de Lyon, 69134 Ecully Cedex, France anton.korniienkodec-lyon.fr, gerard.scorletti@ec-lyon.fr 
that dissipative properties of the leaves are a priori known, a hierarchical analysis approach is to "propagate" them into a new one for the branches. Then the obtained propagated properties can again be propagated, and so on. The analysis boils down to find the propagated properties for the branch from the ones of the leaves. Due to the NP-hardness of robust analysis problem and from a practical point of view, such a propagation cannot be performed exactly since it is inefficient. Nevertheless, as in the case of upper bound on $\mu$, it is possible to perform the propagation in an approximate fashion. Depending on "how good" the propagation approximation is, it will set the "conservatism vs. computation" complexity trade-off. The main purpose of this paper is to add the possibility of setting the propagation precision and thus needed trade-off.

The propagated properties can be viewed as embeddings of the corresponding branch. A first solution to this embedding problem was proposed in [6]. Unfortunately, the embedding introduces some conservatism. It can be reduced by combining different classes of embeddings. The contribution of this paper is to propose new classes of embeddings, in addition to the one of [6], in order to reduce the conservatism. The choice of classes and the number of combined embeddings allows to set the trade-off between conservatism and computation time, which was not possible in [6].

Our solution is based on a separation of graph theorem. First proposed in [15] as a general approach to feedback system analysis, specialized forms were proposed in e.g. [10], [19] for (uncertain) LTI system analysis. Since the $\mu$ upper bound proposed in [8] can be interpreted as a particular application of the separation of graph theorem, this theorem was applied to extend $\mu$-analysis to time-delay [19] or to time-varying/nonlinear [14] systems, to reduce the conservatism of the $\mu$ upper bound [20], to cite a few. In this paper, we reveal another interesting application of this powerful theorem.

Section II begins with definitions and fundamental properties which are used afterwards. It then precises the uncertain large-scale system under consideration in the proposed approach. Due to the size constraint, some proofs will not be presented in the paper but can be found in [5]. Section III proposes several dissipative properties that can be used practically. A numerical example on a PLL network is performed in Section IV. Section V concludes the paper.

Notations. $\overline{\mathbf{R}}$ denotes $\mathbf{R} \cup\{-\infty,+\infty\} . M_{\mathbb{R}}$ and $M_{\mathbb{I}}$ stands for the real and imaginary parts of $M$. For several matrices $M_{i}, i=1, \ldots, n, \mathbf{b d i a g}_{i}\left(M_{i}\right)$ denotes the block diagonal matrix composed of $M_{i}$. $\mathbf{R} \mathbf{H}_{\infty}$ (respectively $\mathbf{R L}_{\infty}$ ) denotes the set of matrices of stable (resp. non causally stable) rational transfer functions. Moreover, we consistently denote uncertainties by $\Delta$ and interconnections by $M$. The set $\Delta$ is referred to as the uncertainty set. We denote by $\boldsymbol{\Delta} \star M$ the set $\{\Delta \star M, \Delta \in \boldsymbol{\Delta}\}$, with $\star$ standing for the Redheffer star product. This set is also referred to as an uncertain system. For the uncertain system $\Delta \star M$, we further denotes the interconnection's partitioning of appropriate dimension by $M=\left[\begin{array}{cc}A & B \\ C & D\end{array}\right]$. Finally, we denote by $\mathcal{L}\left(M, \Phi_{11}, \Phi_{12}, \Phi_{22}, X, Y, Z\right)$ the matrix

$$
\left[\begin{array}{c}
M \\
I
\end{array}\right]^{*}\left[\begin{array}{cc|cc}
-\Phi_{22} & 0 & -\Phi_{12}^{*} & 0 \\
0 & X & 0 & Y \\
\hline-\Phi_{12} & 0 & -\Phi_{11} & 0 \\
0 & Y^{*} & 0 & Z
\end{array}\right]\left[\begin{array}{c}
M \\
I
\end{array}\right]
$$




\section{ApPROACH FOR HiERARCHICAL ANALYSis OF PERFORMANCES}

\section{A. Definitions and preliminaries}

An uncertain system is modeled as an interconnection $\Delta \star M$ with $\Delta \in \Delta$. Introducing the internal signals and using the Fourier transform, we obtain:

$$
\begin{aligned}
p(j \omega) & =\Delta(j \omega) q(j \omega) \\
{\left[\begin{array}{l}
q(j \omega) \\
z(j \omega)
\end{array}\right] } & =\left[\begin{array}{ll}
A(j \omega) & B(j \omega) \\
C(j \omega) & D(j \omega)
\end{array}\right]\left[\begin{array}{l}
p(j \omega) \\
w(j \omega)
\end{array}\right] .
\end{aligned}
$$

Along with this definition, the following is assumed.

Assumption 2.1: $\boldsymbol{\Delta}$ is a bounded and connected subset of $\mathbf{R H}_{\infty}$ and $M$ belongs to $\mathbf{R H}_{\infty}$.

Definition 2.1: An uncertain system $\Delta \star M$ is said to be stable if for any $\Delta \in \Delta$, the system $\Delta \star M$ is stable.

In this section, dissipative properties are used.

Definition 2.2: Let $X(j \omega), Y(j \omega)$ and $Z(j \omega)$ be 3 transfer functions of $\mathbf{R L}_{\infty}$ such that $X(j \omega)=X(j \omega)^{*}$ and $Z(j \omega)=Z(j \omega)^{*}$. Then,

1) a system $H$ is said to be $\{X(j \omega), Y(j \omega), Z(j \omega)\}$ dissipative if for any $\omega \in \overline{\mathbf{R}}$ and for any non null $\left[z^{T}(j \omega)\right.$, $\left.w^{T}(j \omega)\right]^{T}$ verifying $z(j \omega)=H(j \omega) w(j \omega)$ :

$$
\left[\begin{array}{l}
z(j \omega) \\
w(j \omega)
\end{array}\right]^{*}\left[\begin{array}{cc}
X(j \omega) & Y(j \omega) \\
Y(j \omega)^{*} & Z(j \omega)
\end{array}\right]\left[\begin{array}{l}
z(j \omega) \\
w(j \omega)
\end{array}\right]>0 ;
$$

2) an uncertainty set $\boldsymbol{\Delta}$ (or an uncertain system $\boldsymbol{\Delta} \star M)$ is said to be $\{X(j \omega), Y(j \omega), Z(j \omega)\}$ dissipative if for any $\Delta \in \Delta, \Delta$ (or resp. the system $\Delta \star M)$ is $\{X(j \omega), Y(j \omega), Z(j \omega)\}$ dissipative.

A fundamental property is now given. It states that new dissipativity properties can be generated from original ones defining a set of linearly parametrized dissipative properties. Let us denote it $\boldsymbol{\Phi}(j \omega)$. For ease of notation, it is stated for certain systems, the extension to uncertain systems is straightforward.

Corollary 2.1: Let $H_{i}$ be $\left\{X_{i k}(j \omega), Y_{i k}(j \omega), Z_{i k}(j \omega)\right\}$ dissipative, $i=1, \ldots, m$ and $k=1, \ldots, n$. Then for any $\tau_{i k}(\omega)>0, H=\operatorname{bdiag}_{i}\left(H_{i}\right)$ is $\left\{\operatorname{bdiag}_{i}\left(\sum_{k} \tau_{i k}(\omega) X_{i k}(j \omega)\right), \operatorname{bdiag}_{i}\left(\sum_{k} \tau_{i k}(\omega) Y_{i k}(j \omega)\right), \operatorname{bdiag}_{i}\left(\sum_{k} \tau_{i k}(\omega) Z_{i k}(j \omega)\right)\right\}$ dissipative.

\section{B. Hierarchical system description and proposed approach}

From [16], a large-scale system is described by a tree as illustrated in Fig. 1 where a hierarchical structure arises naturally. Each branch of the tree is assigned an index. A branch, say $i$, is a two-way channel through which a signal $w_{i}$ (the input) ascends and another signal $z_{i}$ (the output) descends. The tree obtained by cutting branch $i$ and retaining everything connected above is an uncertain system called $\boldsymbol{T}_{\boldsymbol{i}}$ with input $w_{i}$ and output $z_{i}$. If a tree $\boldsymbol{T}_{\boldsymbol{i}}$ has other branches besides branch $i$ then there is a single node denoted $M_{i}$ from which other branches ascend. 


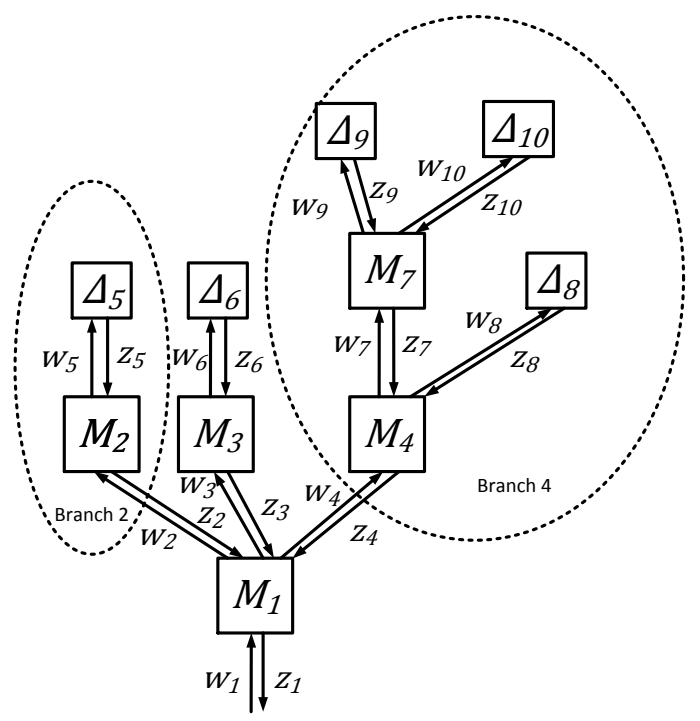

Fig. 1. Uncertain linear large scale system

If branch $i$ is the only branch in the tree, then $\boldsymbol{T}_{\boldsymbol{i}}$ is called a leaf and is denoted by $\boldsymbol{\Delta}_{i}$. Each $M_{i}$ and $\Delta_{i}$ is an LTI system. Furthermore, each leaf $\boldsymbol{\Delta}_{i}$ is uncertain but its dissipative properties are a priori known.

Assumption 2.2: Each $\boldsymbol{\Delta}_{i}$ is a bounded and connected subset of $\mathbf{R} \mathbf{H}_{\infty}$ and each $M_{i}$ belongs to $\mathbf{R} \mathbf{H}_{\infty}$.

Assumption 2.3: All $\boldsymbol{\Delta}_{i}$ are elementary uncertainty sets: there exist a priori known $X_{i k}(j \omega), Y_{i k}(j \omega), Z_{i k}(j \omega)$ such that each $\boldsymbol{\Delta}_{i}$ is $\left\{X_{i k}(j \omega), Y_{i k}(j \omega), Z_{i k}(j \omega)\right\}$ dissipative.

The first assumption is the counterpart of Assumption 2.1 and examples of elementary uncertainty sets along with their dissipative properties can be found in [8], [18], [20].

The proposed approach for the performance analysis of a hierarchical system as described in the introduction is based on a recursive application of the following propagation ${ }^{1}$ of dissipativity properties [17], [18], [21].

Theorem 2.1: Let $\Delta$ be bounded and connected. Then the uncertain system $\Delta \star M$ is stable and $\{X(j \omega), Y(j \omega), Z(j \omega)\}$ dissipative if and only if:

1) there exists $\Delta_{0} \in \Delta$ such that $\Delta_{0} \star M$ is stable;

2) there exist 3 transfer functions $\Phi_{11}(j \omega), \Phi_{12}(j \omega)$ and $\Phi_{22}(j \omega)$ of $\mathbf{R L}_{\infty}$, with $\Phi_{11}(j \omega)=\Phi_{11}(j \omega)^{*}$ and $\Phi_{22}(j \omega)=\Phi_{22}(j \omega)^{*}$, such that the uncertainty set $\boldsymbol{\Delta}$ is $\left\{\Phi_{11}(j \omega), \Phi_{12}(j \omega), \Phi_{22}(j \omega)\right\}$ dissipative and such that $\forall \omega \in \overline{\mathbf{R}}$,

$$
\mathcal{L}\left(M(j \omega), \Phi_{11}(j \omega), \Phi_{12}(j \omega), \Phi_{22}(j \omega), X(j \omega), Y(j \omega), Z(j \omega)\right)>0
$$

Condition 1 is generally viewed as an assumption which is verified beforehand on the nominal system. This assumption is hopefully very mild and is even weaker to the one traditionally assumed in $\mu$-analysis [22] i.e. $\Delta_{0}=0$. In our case, it is an important fact from a practical point of view: the theorem is recursively applied, the uncertainty set (the previous branches) does not necessarily contain 0 .

\footnotetext{
${ }^{1}$ The term propagation is kept in reference to [16] even if the meaning is slightly different.
} 
The fact that the uncertainty set $\boldsymbol{\Delta}$ is $\left\{\Phi_{11}(j \omega), \Phi_{12}(j \omega), \Phi_{22}(j \omega)\right\}$ dissipative in condition 2 can also be verified a priori using Assumption 2.3 and the set of linearly parametrized dissipativity properties $\boldsymbol{\Phi}(j \omega)$ as defined by Corollary 2.1. This assumption allows to increase the efficiency of the approach. It is possible to find them directly, and thus suppress it, as in [9] for instance.

As a consequence, Theorem 2.1 boils down in practice to verify condition (2).

Corollary 2.2: Let $\boldsymbol{\Delta}$ be bounded and connected and let $\boldsymbol{\Phi}(j \omega)$ be a set such that for any $\left(\Phi_{11}(j \omega), \Phi_{12}(j \omega), \Phi_{22}(j \omega)\right) \in$ $\boldsymbol{\Phi}(j \omega)$, the uncertainty set $\boldsymbol{\Delta}$ is $\left\{\Phi_{11}(j \omega), \Phi_{12}(j \omega), \Phi_{22}(j \omega)\right\}$ dissipative.

Then the uncertain system $\Delta \star M$ is stable and $\{X(j \omega), Y(j \omega), Z(j \omega)\}$ dissipative if there exists $\left(\Phi_{11}(j \omega), \Phi_{12}(j \omega)\right.$, $\left.\Phi_{22}(j \omega)\right) \in \boldsymbol{\Phi}(j \omega)$ such that $\forall \omega \in \overline{\mathbf{R}}(2)$ holds.

When two uncertain systems $\boldsymbol{\Delta}_{1} \star M_{1}$ and $\boldsymbol{\Delta}_{2} \star M_{2}$ are homogenous, then they share the same dissipative properties: if $M_{1}=M_{2}$ and $\boldsymbol{\Delta}_{1}=\boldsymbol{\Delta}_{2}$, then $\boldsymbol{\Delta}_{1} \star M_{1}$ is $\{X(j \omega), Y(j \omega), Z(j \omega)\}$ dissipative if and only if $\boldsymbol{\Delta}_{2} \star M_{2}$ is $\{X(j \omega), Y(j \omega), Z(j \omega)\}$ dissipative. This is the case of the PLL network example of Section IV.

\section{Proposed approach with frequency gridding}

As it is usual for LTI systems, a frequency by frequency approach can be performed without loss of generality [4]. From now on, the frequency is thus set to some value and we manipulate complex matrices. For this reason, the dependency on $j \omega$ is dropped.

Let us now illustrate the use of Corollary 2.2 with the system presented in Fig. 1. First, from the dissipative properties of $\boldsymbol{\Delta}_{9}$ and $\boldsymbol{\Delta}_{10}$ that were assumed to be know a priori, find several dissipative properties of the branch $\boldsymbol{T}_{\mathbf{7}}$ using Corollary 2.2 with $\Delta=\operatorname{bdiag}\left(\Delta_{9}, \Delta_{10}\right)$ and $M=M_{7}$. From these several dissipative properties and from Corollary 2.1, it is possible to find a set $\boldsymbol{\Phi}_{7}=\{X, Y, Z\}$ such that for any $\{X, Y, Z\} \in \mathbf{\Phi}_{7}, \boldsymbol{T}_{\mathbf{7}}$ is $\{X, Y, Z\}$ dissipative. From this set and from the dissipativity properties of $\boldsymbol{\Delta}_{8}$, use again Corollary 2.2 with $\Delta=\operatorname{bdiag}\left(T_{7}, \Delta_{8}\right)$ and $M=M_{4}$ to find dissipative properties of the branch $\boldsymbol{T}_{\mathbf{4}}$, and so on until branch $\boldsymbol{T}_{\mathbf{1}}$ is reached where the dissipativity property is a performance index. The overall trade-off between conservatism and computation time then depends on the number of dissipative properties that are searched for at each step and which is user-defined.

However, this procedure is only possible if $\boldsymbol{T}_{\mathbf{7}}$ and then $\boldsymbol{T}_{\mathbf{4}}, \boldsymbol{T}_{\mathbf{1}}$ etc. are bounded and connected sets. As it is presented in the following lemma, it is indeed the case if the dissipativity properties are well chosen. Typically, it is needed that $X$ is negative definite, i.e. a conic sector property, see Section III-A.

Lemma 2.1: Let $X, Y$ and $Z$ be 3 complex matrices such that $X=X^{*}$ and $Z=Z^{*}$. Assume that $X<0$, then the set of matrices $\{H \mid H$ is $\{X, Y, Z\}$ dissipative $\}$ is convex, and thus connected, and bounded.

Proof: Let us define

$$
H_{c}=-X^{-1} Y \text { and } R^{*} R=Z-Y^{*} X^{-1} Y \text {. }
$$

The dissipativity property of a system $H$ writes then

$$
\left(z-z_{c}\right)^{*}(-X)\left(z-z_{c}\right)<w^{*} R^{*} R w
$$


with $z=H w$ and $z_{c}=H_{c} w$. Or equivalently $\left\|(-X)^{1 / 2}\left(H-H_{c}\right) w\right\|_{2}<\|R w\|_{2}$, which gives

$$
\bar{\sigma}\left((-X)^{1 / 2}\left(H-H_{c}\right) R^{-1}\right)<1
$$

with $\bar{\sigma}$ the maximum singular value. This set corresponds to a ball centered around $H_{c}$ with a weighted norm. It is thus convex and bounded.

\section{Practical Formulation of Dissipativity Propagation}

In this section, we show how to find dissipative properties (referred to as 'propagated' in the introduction) for the uncertain system $\Delta \star M$ from the ones of $\Delta$ : it is the propagation of dissipativity properties. The problem can be stated as follows.

Problem 3.1: Let $\boldsymbol{\Phi}$ be a set such that for any $\left(\Phi_{11}, \Phi_{12}, \Phi_{22}\right) \in \boldsymbol{\Phi}$, the uncertainty set $\boldsymbol{\Delta}$ is $\left\{\Phi_{11}, \Phi_{12}, \Phi_{22}\right\}$ dissipative.

From the set $\boldsymbol{\Phi}$, find $X, Y$ and $Z$ such that the uncertain system $\boldsymbol{\Delta} \star M$ is $\{X, Y, Z\}$ dissipative.

Based on Corollary 2.2, problem 3.1 is solved by the following optimization: find $\left(\Phi_{11}, \Phi_{12}, \Phi_{22}\right) \in \mathbf{\Phi}, X, Y$ and $Z$ such that Corollary 2.2 conditions are satisfied.

Note that this optimization problem parametrizes all the possible propagated properties from the ones of $\Delta$ in $\boldsymbol{\Phi}:$ it is non conservative from a propagation perspective.

In the way the propagation is used, $\Delta$ is either a leaf or a branch. In both case, either due to Assumption 2.3 or Corollary 2.1, the set $\boldsymbol{\Phi}$ is of the form $\left\{\sum_{k} \tau_{k}\left(\Phi_{11 k}, \Phi_{12 k}, \Phi_{22 k}\right)\right\}$ with a priori known $\left(\Phi_{11 k}, \Phi_{12 k}, \Phi_{22 k}\right)$. The optimization problem thus boils down to find $\tau_{i}$ and $X, Y$ and $Z$ such that (2) holds. It is thus an LMI optimization problem, is convex and can be solved efficiently. Corollary 2.2 defines a optimization problem with complex LMI constraints. For computational purpose, they can readily be converted as real LMI constraints [2]. These remarks hold for all the optimization problems involved in this section.

To improve the overall conservatism of the hierarchical analysis, it is interesting to obtain the 'tightest' propagated dissipativity property. It is performed by interpreting the property in geometrical terms. For each geometrical interpretation, a notion of size is defined and one is interested in minimizing this size.

\section{A. Conic sector: $X<0$}

In the case when $X<0$, the dissipativity property can be rewritten as (3) and (4) and defines the same set as a conic sector [16] in which a system $H$ is said to be in the conic sector $(C, P, Q)$, with $C$ the cone center, whenever

$$
\left\|Q^{-1 / 2}(z-C w)\right\|_{2}<\left\|P^{1 / 2} w\right\|_{2} \text { with } z=H w
$$

The link is provided by

$$
Q=-X^{-1}, C=H_{c} \text { and } P=R^{*} R
$$


For a SISO system, the inequality (4) defines a disk of center $z_{c}$ and radius $w^{*}\left(X /\left(R^{*} R\right)\right) w$. More generally, it is an ellipsoid. Indeed, the inequality can be rewritten for real matrices and vectors as

$$
\left[\begin{array}{c}
z_{\mathbb{R}}-z_{c \mathbb{R}} \\
-\left(z_{\mathbb{I}}-z_{c \mathbb{I}}\right)
\end{array}\right]^{T} \mathcal{P}\left[\begin{array}{c}
z_{\mathbb{R}}-z_{c \mathbb{R}} \\
-\left(z_{\mathbb{I}}-z_{c \mathbb{I}}\right)
\end{array}\right]<1
$$

with

$$
\mathcal{P}=\frac{\left[\begin{array}{cc}
-X_{\mathbb{R}} & -X_{\mathbb{I}} \\
X_{\mathbb{I}} & -X_{\mathbb{R}}
\end{array}\right]}{\left[\begin{array}{c}
w_{\mathbb{R}} \\
-w_{\mathbb{I}}
\end{array}\right]^{T}\left[\begin{array}{cc}
\left(R^{*} R\right)_{\mathbb{R}} & \left(R^{*} R\right)_{\mathbb{I}} \\
-\left(R^{*} R\right)_{\mathbb{I}} & \left(R^{*} R\right)_{\mathbb{R}}
\end{array}\right]\left[\begin{array}{c}
w_{\mathbb{R}} \\
-w_{\mathbb{I}}
\end{array}\right]} .
$$

Thus, for a given non null input $w$, the corresponding output signal $\tilde{z}=\left[\begin{array}{ll}z_{\mathbb{R}}^{T} & -z_{\mathbb{I}}^{T}\end{array}\right]^{T}\left(\right.$ with $\tilde{z}_{c}=\left[\begin{array}{ll}z_{c \mathbb{R}}^{T} & -z_{c \mathbb{I}}^{T}\end{array}\right]^{T}$ ) belongs to the ellipsoid

$$
\epsilon_{\mathcal{P}}=\left\{\tilde{z} \mid\left(\tilde{z}-\tilde{z}_{c}\right)^{T} \mathcal{P}\left(\tilde{z}-\tilde{z}_{c}\right)<1\right\}
$$

Definition 3.1: The volume of the ellipsoid $\epsilon_{\mathcal{P}}$ defined by (7) and (6) is defined as

$$
\operatorname{vol}\left(\epsilon_{\mathcal{P}}\right)^{2}=\beta \operatorname{det}\left(\mathcal{P}^{-1}\right)
$$

where $\beta$ is a positive scalar which depends on the size $n_{z}$ of the vector $\tilde{z}-\tilde{z}_{c}$ (see [1]).

We are interested in finding the ellipsoid with the smallest volume for all inputs such that $\|w\|=1$.

Problem 3.2: Let $\boldsymbol{\Phi}$ be a set such that for any $\left(\Phi_{11}, \Phi_{12}, \Phi_{22}\right) \in \boldsymbol{\Phi}$, the uncertainty set $\boldsymbol{\Delta}$ is $\left\{\Phi_{11}, \Phi_{12}, \Phi_{22}\right\}$ dissipative.

From the set $\boldsymbol{\Phi}$, find $X, Y$ and $Z$ such that:

1) the uncertain system $\boldsymbol{\Delta} \star M$ is $\{X, Y, Z\}$ dissipative;

2) they minimize $\max _{\Delta \in \Delta} \max _{\|w\|=1} \operatorname{vol}\left(\epsilon_{\mathcal{P}}\right)^{2}$.

Theorem 3.1 (see [5] for the proof): Problem 3.2 is solved by the following optimization problem: find $\left(\Phi_{11}, \Phi_{12}, \Phi_{22}\right) \in$ $\mathbf{\Phi}, X, Y, Z$ that minimize $\log \left(\operatorname{det}\left(\left[\begin{array}{cc}-X_{\mathbb{R}} & -X_{\mathbb{I}} \\ X_{\mathbb{I}} & -X_{\mathbb{R}}\end{array}\right]^{-1}\right)\right)$ and such that

1) $\mathcal{L}\left(M, \Phi_{11}, \Phi_{12}, \Phi_{22}, X, Y, Z\right)>0$ holds;

2) $\left[\begin{array}{ll}I & 0 \\ 0 & 0\end{array}\right] \geq\left[\begin{array}{cc}Z & Y^{*} \\ Y & X\end{array}\right]$ holds.

This optimization problem is a determinant maximization under linear matrix inequality constraints [24] and is convex.

B. Half Planes: $X=0$

a) Half plane: A dissipativity property with $X=0$ rewrites

$$
\xi^{T}\left[\begin{array}{c}
z_{\mathbb{R}} \\
-z_{\mathbb{I}}
\end{array}\right]-\eta>0
$$


with

$$
\begin{array}{rr}
\xi & =2\left[\begin{array}{cc}
Y_{\mathbb{R}} & Y_{\mathbb{I}} \\
-Y_{\mathbb{I}} & Y_{\mathbb{R}}
\end{array}\right]\left[\begin{array}{c}
w_{\mathbb{R}} \\
-w_{\mathbb{I}}
\end{array}\right], \\
\eta & =-\left[\begin{array}{c}
w_{\mathbb{R}} \\
-w_{\mathbb{I}}
\end{array}\right]^{T}\left[\begin{array}{cc}
Z_{\mathbb{R}} & Z_{\mathbb{I}} \\
-Z_{\mathbb{I}} & Z_{\mathbb{R}}
\end{array}\right]\left[\begin{array}{c}
w_{\mathbb{R}} \\
-w_{\mathbb{I}}
\end{array}\right]
\end{array}
$$

which express that for a given input signal $w$ the output signal $z$ belongs to a half plane defined by the hyperplane:

$$
\left\{\left[\begin{array}{c}
z_{\mathbb{R}} \\
z_{\mathbb{I}}
\end{array}\right] \mid \xi^{T}\left[\begin{array}{c}
z_{\mathbb{R}} \\
-z_{\mathbb{I}}
\end{array}\right]=\eta\right\} .
$$

Where $\xi$ is a vector normal to the hyperplane and $\eta$ is the twice 'signed distance' of the hyperplane to the origin (the dot product of any point of the hyperplane with $\xi$ ).

b) Band: A band is the intersection of two half planes with the same normal direction but opposite sign. The size of a band is defined by the distance between the two parallel hyperplanes.

Definition 3.2: Let $\xi, \eta_{1}$ and $\eta_{2}$ be of appropriate dimensions and define the two half planes $\xi^{T}\left[\begin{array}{c}z_{\mathbb{R}} \\ -z_{\mathbb{I}}\end{array}\right]-\eta_{1}>0$ and $-\xi^{T}\left[\begin{array}{c}z_{\mathbb{R}} \\ -z_{\mathbb{I}}\end{array}\right]-\eta_{2}>0$. The size of the corresponding band is defined by $d_{Y}=\eta_{2}-\eta_{1}$.

As for the conic sector, we are interested in the smallest band for a given direction, that is for a given $Y$ for all inputs such that $\|w\|=1$.

Problem 3.3: Let $\boldsymbol{\Phi}$ be a set such that for any $\left(\Phi_{11}, \Phi_{12}, \Phi_{22}\right) \in \boldsymbol{\Phi}$, the uncertainty set $\boldsymbol{\Delta}$ is $\left\{\Phi_{11}, \Phi_{12}, \Phi_{22}\right\}$ dissipative. Let $Y$ be a matrix of appropriate dimension.

From the set $\boldsymbol{\Phi}$ and $Y$, find $Z_{1}$ and $Z_{2}$ such that:

1) the uncertain system $\boldsymbol{\Delta} \star M$ is $\left\{0, Y, Z_{1}\right\}$ dissipative;

2) the uncertain system $\boldsymbol{\Delta} \star M$ is $\left\{0,-Y, Z_{2}\right\}$ dissipative;

3) they minimize $\max _{\Delta \in \Delta} \max _{\|w\|=1} d_{Y}$.

Theorem 3.2 (see [5] for the proof): Problem 3.3 is solved by the following optimization problem: find $\left(\Phi_{111}, \Phi_{121}, \Phi_{221}\right) \in$ $\mathbf{\Phi}$ and $\left(\Phi_{211}, \Phi_{212}, \Phi_{222}\right) \in \mathbf{\Phi}, Z_{1}, Z_{2}$ that minimize $d$ such that

1) $\mathcal{L}\left(M, \Phi_{111}, \Phi_{112}, \Phi_{122}, 0, Y, Z_{1}\right)>0$ holds;

2) $\mathcal{L}\left(M, \Phi_{211}, \Phi_{212}, \Phi_{222}, 0,-Y, Z_{2}\right)>0$ holds;

3) $Z_{1}+Z_{2} \leq d I$ holds;

This optimization problem is a minimization of a linear cost under linear matrix inequality constraints [1] and is convex.

It is then possible to generate several bands with different normals as performed in the example of Section IV. It is also possible to search for the direction of the band by letting $Y$ to be free. 


\section{PLL NETWORK EXAMPLE}

Let us now consider a numerical example of hierarchical performance analysis of an uncertain large-scale system. One takes as an example the performance analysis of the active clock distribution network from [11] subject to technological dispersions. An active clock distribution network is composed of $N=16$ mutually synchronized Phase-Locked-Loops (they constitute branches of the tree) delivering the clock signals to the chip. Please note that the PLL is a Single Input Single Output (SISO) system. To be able to synchronize the PLLs exchange the information on their relative phase through the interconnection network and the phase detectors. This example is particularly well adapted as the performance is measured in frequency domain.

\section{A. PLL network description}

Since the principal aim of the system is the synchronization, the PLLs are homogeneous i.e. have a common interconnection and the same uncertainty set. Of course, during the manufacturing process, there are inevitable technological dispersions which can be represented in the form of parametric uncertainties belonging to the same set. We have thus $\forall i \in\{1, \ldots, N\}$ :

$$
T_{i}(j \omega)=\frac{k_{i}\left(j \omega+a_{i}\right)}{-\omega^{2}+k_{i} j \omega+k_{i} a_{i}}
$$

where $k_{i}, a_{i}$ are the real uncertain parameters defined as $k_{i} \in\left(0.76 \cdot 10^{4}, 6.84 \cdot 10^{4}\right)$ and $a_{i} \in(91.1,273.3)$ and $\omega$ is the current frequency defined by gridding.

The exchange of information between the PLLs in the network is modeled by an interconnection matrix $M_{n e t}$ as defined in [5] (see equation (8)). In this example, the transfer function between external signals $w$ and $z$ expresses the performance of the global PLL network and namely its ability to synchronize with periodic reference signal $w$. This reference signal is represented by its phase so that the PLL network has to track a ramp (see [11], [13] for more details).

\section{B. Hierarchical analysis set up}

The proposed hierarchical analysis approach is applied in two steps for this PLL network:

1) obtain dissipativity properties of each individual PLL, each PLL being a branch. Since the PLLs are homogeneous, the dissipativity properties obtained for one PLL is valid for the others as well;

2) obtain the performance of the overall network thought the interconnection of the 16 PLL branches and $M_{n e t}$.

Individual PLL: Each PLL can be readily written in the form of an interconnection, which leads after normalization of the uncertainties to:

$$
T_{i}(j \omega)=\Delta_{i} \star M_{P L L}, \Delta_{i} \in \Delta
$$

with $\Delta$ of the form

$$
\left\{\left[\begin{array}{cc}
\delta k_{i} & 0 \\
0 & \delta a_{i}
\end{array}\right], \delta=\left[\begin{array}{c}
\delta k_{i} \\
\delta a_{i}
\end{array}\right] \in \mathbb{R}^{2},\|\delta\|_{\infty} \leq 1\right\} .
$$

It is a standard elementary uncertainty set (the leaves) representing parametric uncertainties. The dissipativity property of the uncertainty set $\boldsymbol{\Phi}$ can then be chosen in the form of $D$ - $G$ scaling as for classical $\mu$-analysis [8]. 


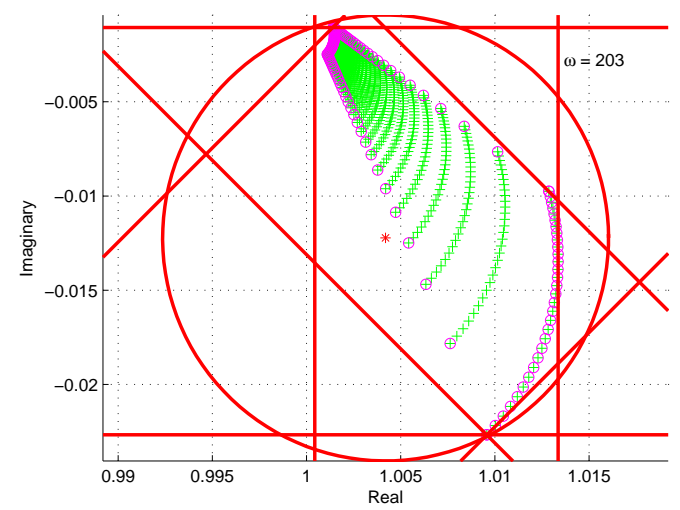

Fig. 2. Dissipativity properties of a PLL viewed as embeddings

Additionally the $L$ scaling was introduced in [20] to reduce the conservatismproposing $D-G-L$ scaling which will be used hereafter.

As for the dissipativity properties of the PLL itself, we chose:

- a conic sector alone (for comparison with the result obtained in [6]);

- a conic sector and 4 bands (vertical, horizontal, and with a slope of $+/-45 \mathrm{deg}$ ): $Y \in\{1, j, 1+j, 1-j\}$. This choice has been made a priori, without particular knowledge on a PLL frequency response.

Please note that for this choice of dissipativity properties the Lemma 2.1 and hence proposed hierarchical analysis approach can be applied since at least one dissipativity property is defined with $X<0$.

Network performance: The network performance is measured by its frequency response magnitude bound. The dissipativity property is thus chosen of the form $\left[\begin{array}{cc}-I & 0 \\ 0 & \gamma^{2} I\end{array}\right]$ which is a particular conic sector.

\section{Results}

Individual PLL: For illustration purpose, Fig. 2 displays the obtained dissipativity properties of a PLL for a fixed frequency $\omega=203 \mathrm{rad} / \mathrm{sec}$. The red circle (the red star is its center) and lines represent the embeddings where as the green stars and purple circles represent the PLL frequency response at $\omega=203 \mathrm{rad} / \mathrm{sec}$ for some values of the uncertainties.

Network performance: We are now interested in the performance of the PLLs network displayed in Fig. 3 while Table I displays the characteristics of the different analysis (the number in parenthesis for the hierarchical analysis columns is a comparison with the $\mu$-analysis results i.e. direct approach of [8].

All the analysis reveal that the PLL network is able to track a ramp as the slope of frequency response magnitude at low frequencies is $40 \mathrm{~dB} / \mathrm{dec}$. Table I illustrates the trade-off between conservatism and computation time that can be set by the user with the hierarchical analysis approach: when using the conic sector alone, the result is conservative but is obtained really quickly; when using the conic sector with the bands, the result is much less conservative but is obtained in much more time. For this last hierarchical setup, the difference in the maximal peak 


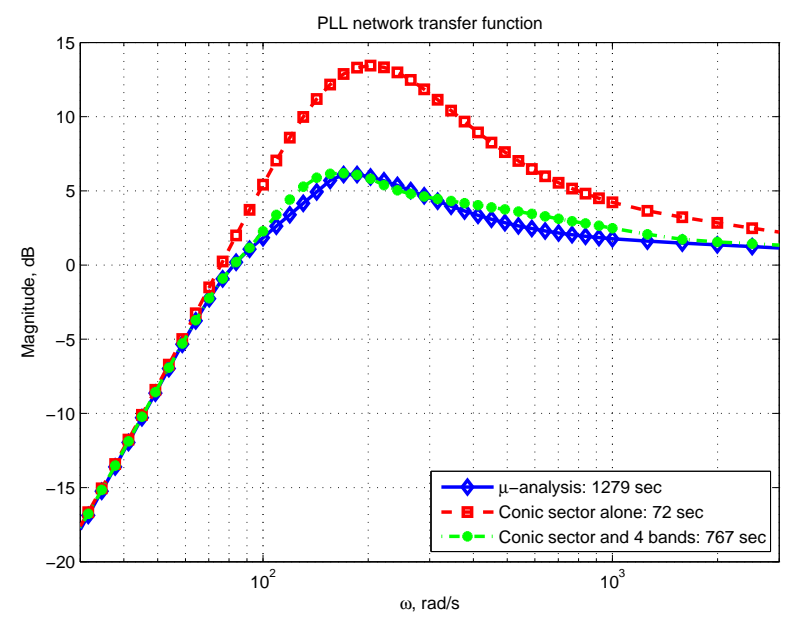

Fig. 3. Performance of the PLLs network

TABLE I

CHARACTERISTICS OF THE ANALYSES

\begin{tabular}{|c|c|c|c|}
\hline & \multicolumn{2}{|c|}{ Hierarchical analysis } & $\mu$-analysis \\
& Conic sector & Conic sector & \\
& alone & and 4 bands & \\
\hline Maximum peak & $13.5 \mathrm{~dB}(+7.4)$ & $6.2 \mathrm{~dB}(+0.1)$ & $6.1 \mathrm{~dB}$ \\
Computation time & $72 \mathrm{sec}(6 \%)$ & $767 \mathrm{sec}(60 \%)$ & $1279 \mathrm{sec}$ \\
\hline
\end{tabular}

value with $\mu$-analysis is $+0.1 \mathrm{~dB}$, that corresponds to $1.2 \%$ of ratio, which is negligible; the result was obtained in $60 \%$ of the time needed for $\mu$-analysis.

\section{CONClusion}

In this paper, a hierarchical analysis approach has been proposed for the performance of uncertain large-scale systems. It relies on the propagation of dissipativity properties of sub-systems through an interconnection; this propagation result is recursively applied leading to a multi steps analysis. The aim is to propose a trade-off adapted to these large-scale systems when a one-step approach as $\mu$-analysis can lead to a large computation time. A numerical example on a PLL network illustrated the new achieved trade-off.

Further work directions are:

- find other dissipative properties that can be used. We think to a cone as proposed in [23] for instance;

- further assess the achieved trade-off for other examples, especially for Multi Input Multi Output (MIMO) ones;

- consider frequency ranges instead of frequency points in order to avoid possible frequency gridding problems

- assess the evolution of the achieved trade-off in function of the dissipativity properties used. 


\section{REFERENCES}

[1] S. Boyd, L. El Ghaoui, E. Feron, and V. Balakrishnan. Linear Matrix Inequalities in Systems and Control Theory, volume 15 of Studies in Appllied Mathematics. SIAM, Philadelphia, USA, June 1994.

[2] S. Boyd and L. El Ghaoui. Method of centers for minimizing generalized eigenvalues. Linear Algebra and Applications, special issue on Numerical Linear Algebra Methods in Control, 188:63-111, July 1993.

[3] R. D. Braatz, P. M. Young, J. C. Doyle, and M. Morari. Computational complexity of $\mu$ calculation. IEEE Trans. Aut. Control, AC39(5):1000-1002, May 1994.

[4] Y.-S. Chou, A.L. Tits, and V. Balakrishnan. Stability multipliers and $\mu$ upper bounds: connections and implications for numerical verification of frequency domain conditions. IEEE Trans. Aut. Control, 44(5):906-913, 1999.

[5] M. Dinh, A. Korniienko, and G. Scorletti. Convex hierarchical analysis for the performances of uncertain large-scale systems. Technical report, Laboratoire Ampère, Ecole Centrale de Lyon, 2013.

[6] M. Dinh, A. Korniienko, and G. Scorletti. Embedding of uncertainty propagation: Application to hierarchical performance analysis. In IFAC Joint Conference, 5th Symposium on System Structure and Control, pages 190-195, Grenoble, France, February 2013.

[7] J.C. Doyle. Analysis of feedback systems with structured uncertainties. IEE Proc., 129-D(6):242-250, November 1982.

[8] M. K. H. Fan, A. L. Tits, and J. C. Doyle. Robustness in the presence of mixed parametric uncertainty and unmodeled dynamics. IEEE Trans. Aut. Control, 36(1):25-38, January 1991.

[9] E. Feron, P. Apkarian, and P. Gahinet. Analysis and synthesis of robust control systems via parameter-dependent lyapunov functions. IEEE Trans. Aut. Control, 41(7):1041-1046, July 1996.

[10] T. Iwasaki and S. Hara. Well-posedness of feedback systems: Insights into exact robustness analysis and approximate computations. IEEE Trans. Aut. Control, 43:619-630, 1998.

[11] A. Korniienko, G. Scorletti, E. Colinet, and E. Blanco. Control law design for distributed multi-agent systems. Technical report, Laboratoire Ampère, Ecole Centrale de Lyon, 2011.

[12] A. Korniienko, G. Scorletti, E. Colinet, and E. Blanco. Approche de la performance relative pour la commande de systèmes de grande dimension. In Conférence Internationale Francophone d'Automatique, Grenoble, June 2012. to be appear.

[13] A. Korniienko, G. Scorletti, E. Colinet, E. Blanco, J. Juillard, and D. Galayko. Control law synthesis for distributed multi-agent systems: Application to active clock distribution networks. In Proc. American Control Conf., pages 4691- 4696, San Francisco, June 2011.

[14] A. Megretski and A. Rantzer. System analysis via integral quadratic constraints. IEEE Trans. Aut. Control, 42(6):819-830, June 1997.

[15] M. G. Safonov. Stability and Robustness of Multivariable Feedback Systems. MIT Press, Cambridge, 1980.

[16] M. G. Safonov. Propagation of conic model uncertainty in hierarchical systems. IEEE Trans. Circuits and Systems, pages 388-396, June 1983.

[17] C.W. Scherer. LPV control and full block multipliers. Automatica, 73:361-375, 2001.

[18] G. Scorletti. Robustness analysis with time delays. In IEEE, editor, IEEE Conf. Decision and Control, pages 3824-3829, San Diego, California, December 1997.

[19] G. Scorletti. A more praticle formulation for robustness analysis. In IFAC Conference on System Structure and Control, Nantes, France, July 1998.

[20] G. Scorletti, X. Bombois, M. Barenthin, and V. Fromion. Improved efficient analysis for systems with uncertain parameters. In Proc. IEEE Conf. on Decision and Control, pages 5038-5043, New Orleans, dec. 2007.

[21] G. Scorletti and L. EL Ghaoui. Improved LMI conditions for gain-scheduling and related control problems. Int. J. Robust and Nonlinear Control, 8(10):845-877, August 1998.

[22] S. Skogestad and I. Postlethwaite. Multivariable Feedback Control, Analysis and Design. John Wiley and Sons Chischester, 2005.

[23] A.L. Tits, V. Balakrishnan, and L. Lee. Robustness under bounded uncertainty with phase information. IEEE Trans. Aut. Control, 44(1), 1999.

[24] Lieven Vandenberghe, Stephen Boyd, and Shao-Po Wu. Determinant maximization with linear matrix inequality constraints. SIAM Journal on Matrix Analysis and Applications, 19:499-533, 1996. 